Comment. Math. Helv. 74 (1999) 238-247

$0010-2571 / 99 / 020238-10 \$ 1.50+0.20 / 0$
(C) 1999 Birkhäuser Verlag, Basel

Commentarii Mathematici Helvetici

\title{
Covering degrees are determined by graph manifolds involved
}

Fengchun Yu and Shicheng Wang

\begin{abstract}
W.Thurston raised the following question in 1976: Suppose that a compact 3manifold $M$ is not covered by (surface) $\times S^{1}$ or a torus bundle over $S^{1}$. If $M_{1}$ and $M_{2}$ are two homeomorphic finite covering spaces of $M$, do they have the same covering degree?

For so called geometric 3-manifolds (a famous conjecture is that all compact orientable 3-manifolds are geometric), it is known that the answer is affirmative if $M$ is not a non-trivial graph manifold.

In this paper, we prove that the answer for non-trivial graph manifolds is also affirmative. Hence the answer for the Thurston's question is complete for geometric 3-manifolds. Some properties of 3-manifold groups are also derived.
\end{abstract}

Mathematics Subject Classification (1991). 15A18, 15A24, 20F32, 57M10, 57M25.

Keywords. Graph manifold, covering degree, B-matrix, H-matrix.

\section{Introduction}

Definition 1. A 3-manifold $M$ is said to have Property $C$ if, whenever $M_{1}, M_{2}$ are homeomorphic finite covering spaces of $M$, the degrees of the coverings are the same. It has Property $C_{r}$ if the above is true for all regular coverings. (Noted that $M$ has Property $C$ if and only if it has Property $C_{r}$ [WW, Lemma 2.1].)

We are mainly interested in the following problem of W.Thurston raised in 1976.

$[\mathrm{K}$, problem 3.16 (a)] Which 3-manifolds have Property $C$ ? In particular suppose that $M$ is not covered by (surface) $\times S^{1}$ or a torus bundle over $S^{1}$, does $M$

A comment from the second author: The first author was passed away in 1995, at the age of 23 as a graduate student, and had four joint papers by then. I felt deeply sorry for his untimely death. The authors would like to thank B. Jiang, W. Tang, Y. Wu and G. Yuan for their helps. The work is supported by outstanding Youth fellowship of NSFC. 
has property $C$ ?

Definition 2. A compact, connected, orientable 3-manifold is said to be geometric if it is a Seifert manifold, or a hyperbolic manifold, or a Haken manifold, or a connected sum of such manifolds.

The fundamental conjecture in 3-manifold theory is that all compact, connected, orientable 3 -manifolds are geometric. It is known that if $M$ is covered by either (surface) $\times S^{1}$ or a torus bundle over $S^{1}$, then it does not have Property $C$. Actually such manifolds admit non-trivial self-coverings [WW, Theorem 8.6]. Therefore we will concentrate on 3 -manifolds in the class $\mathcal{G}$ defined below.

Notation. We use $\mathcal{G}$ to denote the set of all geometric 3 -manifolds which are not covered by either (surface) $\times S^{1}$ or a torus bundle over $S^{1}$.

Conjecture 1. Every 3-manifold $M$ in $\mathcal{G}$ has Property $\mathrm{C}$.

By using Gromov norm and Kneser-Milnor sphere decomposition theorem, it is proved that Conjecture 1 is true if $M$ is not a non-trivial graph manifold [WW, Theorem 2.5]. So the problem is reduced to the case of non-trivial graph manifolds.

Several partial results on property $C$ of non-trivial graph manifolds had been obtained recently. There are three different covering invariants of graph manifolds introduced by S.C.Wang and Y.Q.Wu [WW], by J.Luecke and Y.Q.Wu [LW] and by W.D. Neumann [N]. So a graph manifold has property $C$ if one of those covering invariants is non-zero. Unfortunately all those three covering invariants are zero for some non-trivial graph manifolds. It is also proved that a non-trivial graph manifold has property $C$ if $M$ has at most three vertex manifolds [WW] or if $M$ is a knot complement in $S^{3}[\mathrm{LW}]$.

In this paper we prove that non-trivial graph manifolds have property $C$. Hence a geometric manifold has Property $C$ if and only if it is not covered by either (surface) $\times S^{1}$ or a torus bundle over $S^{1}$.

There are four sections after the introduction. In $\S 1$, we review the $B$-matrix $B(M)$ for a non-trivial graph manifold $M$ defined by S.C. Wang and Y.Q.Wu [WW] and its properties, which will be the start point of our further approach. In $\S 2$, we introduce a $H$-matrix $H(M)$ for a non-trivial graph manifold $M$, and then deduce a matrix equation which expresses the covering degree by $B$-matrix and $H$ matrix (Theorem 3). In $\S 3$, we prove our main result: non-trivial graph manifolds have Property $C$ (Corollary 5). Some results about maximum eigenvalues of nonnegative matrices are used in the proof. In $\S 4$, we prove that for a closed geometric 3-manifold $M, \pi_{1}(M)$ have property $I$, that is any two isomorphic subgroups of $\pi_{1}(M)$ have the same index (may be infinite), if and only if $M$ have property $C$ and $M$ is irreducible. This result generalizes the earlier results of F. Gonzales-Acuna and W. Whitten $[\mathrm{GW}]$ and of [WW] on the cohopficity of 3-manifold groups. 
Remark 1. A non-orientable 3-manifold has Property $C$ if and only if its orientable double cover has property $C$.

\section{Preliminaries}

For readers' convenience, we review a matrix invariant defined in [WW] in this section. The notions which are not explained are standard, see $[\mathrm{J}]$ and $[\mathrm{S}]$.

For a surface $S$ in a 3-manifold $M$, we use $N(S)$ to denote the regular neighborhood of $S$ and $\eta(S)$ to denote the interior of $N(S)$.

Suppose $M$ is a prime orientable 3-manifold with boundary (possibly empty) a union of tori. Then the torus decomposition of Jaco-Shalen and Johannson cut $M$ open along a minimum collection of embedded incompressible tori $\mathcal{T}$, the JSJ-surface, into a collection of simple manifolds and Seifert manifolds.

Definition 3. A geometric 3-manifold $M$ is called a graph manifold if the JSJsurface $\mathcal{T}$ is not empty and $\mathcal{T}$ cuts $M$ into Seifert manifold pieces. A graph manifold is non-trivial if it is not covered by a torus bundle over $S^{1}$. (see [WW, $\left.\S 3\right]$. Noted a graph manifold is non-trivial if and only if it belongs to $\mathcal{G}$ ).

Now consider a non-trivial graph manifold $M$. In [WW] a new decomposing surface $\varphi$ of $M$ is defined which consists of those tori in JSJ surface $\mathcal{T}$ which do not bound twisted $I$-bundles, and those central Klein bottles of twisted $I$-bundles bounded by tori in $\mathcal{T}$. If $M$ is a graph manifold in $\mathcal{G}$, then the decomposing surface exists and is unique up to ambient isotopy [WW, Lemma 3.1].

Comparing with $\mathcal{T}$, the new decomposition surface $\varphi$ has two advantages:

Lemma 1. (a) $M-\eta(\varphi)$ consists of Seifert fiber spaces with negative Euler characteristic orbifolds [WW, Lemma 3.2], therefore each Seifert manifold piece of $M$ admits a unique Seifert fibration up to isotopy [S, Theorem 3.9].

(b) Suppose $\phi: \tilde{M} \rightarrow M$ is a finite covering between graph manifolds. If $\varphi$ is a non-empty decomposing surface of $M$, then $\tilde{\varphi}=\phi^{-1}(\varphi)$ in $\tilde{M}$ is a decomposing surface for $\tilde{M}[W W$, Lemma 4.4].

Suppose $M$ is a non-trivial graph manifold and has the decomposing surface $\varphi$ just defined. We define a graph $\Gamma(M)$ as follows: to each component $N_{i}$ of $M-\eta(\varphi)$ a vertex $v_{i}$ is assigned, and to each component $S_{j}$ of $\varphi$ an edge $e_{j}$ is assigned, so that

(1) if $S_{j}$ is a torus, and $\partial N\left(S_{j}\right)$ has one component in each of $N_{i}$ and $N_{k}(i$ may be equal to $k$ ), then $e_{j}$ has endpoints on $v_{i}$ and $v_{k}$;

(2) if $S_{j}$ is a Klein bottle and $\partial N\left(S_{j}\right)$ is in $N_{i}$, then $e_{j}$ has both endpoints on $v_{i}$.

We define a 'weight' for each vertex or edge of $\Gamma(M)$ as follows. If $v_{i}$ is a vertex 
of $\Gamma(M)$ corresponding to a component $N_{i}$ of $M-\eta(\varphi)$, let the weight $x_{i}=x\left(v_{i}\right)$ be the Euler characteristic of the orbifold of $N_{i}$. If $e$ is an edge corresponding to a surface $S$ in $\varphi$, let the weight $\Delta(e)$ be the fiber intersection number $\Delta(S)$. The weights of vertices are negative rational numbers by Lemma 1 (a), and the weights of edges are all positive integers ([WW, Lemma 4.3]). Since the decomposing surface and the Seifert fibrations on the pieces are unique, this weighted graph is well defined, and is an invariant of $M$.

For each edge $e$ in the graph $\Gamma(M)$, define

$$
\alpha(e)=\frac{1}{\Delta(e) x_{i} x_{j}} .
$$

For all $i, j$, define

$$
a_{i j}=a_{i j}(\Gamma(M))= \begin{cases}\sum\left\{\alpha(e) \mid e \text { has the endpoints on } v_{i} \text { and } v_{j}\right\} \\ 0 \quad \text { if there is no such e. }\end{cases}
$$

To simplify the formulas, we define

$$
b_{i j}= \begin{cases}a_{i j} & \text { if } i \neq j, \\ 2 a_{i j} & \text { if } i=j .\end{cases}
$$

Define $\tilde{b}_{p q}$ in the same way.

Let $B=B(M)=\left(b_{i j}\right)$. The matrix $B$ depends only on $\Gamma(M)$ and the indexing of its vertices, so up to simultaneous permutations of rows and columns it is well defined. Clearly $B$ is a non-negative symmetric matrix.

Definition 4. $\Gamma=\Gamma(M)$ is called the weighted graph of $M$, and $B=B(M)$ is called the $B$-matrix of $M$, for a non-trivial graph manifold $M$.

Consider a regular covering map $\phi: \tilde{M} \rightarrow M$. By Lemma 1 (b), $\phi$ can induce a map on the graphs $\phi_{\#}: \Gamma(\tilde{M}) \rightarrow \Gamma(M)$ defined in the following way. A vertex $\tilde{v_{p}}$ is mapped to $v_{i}$ if the corresponding component $\tilde{N}_{p}$ in $\tilde{M}-\eta(\tilde{\varphi})$ is mapped to $N_{i}$, and an edge $\tilde{e_{g}}$ is mapped to $e_{h}$ if the corresponding surface $\tilde{S_{g}}$ covers $S_{h}$.

For each vertex $v_{i}$ in $\Gamma(M)$, define

$$
I_{i}=\left\{p \mid \phi\left(\tilde{v}_{p}\right)=v_{i}\right\}
$$

and use $\left|I_{i}\right|$ to denote the number of elements in $I_{i}$.

The result below is the start point for our approach.

Theorem 1. ([WW, Theorem 6.1]) Suppose $\phi: \tilde{M} \rightarrow M$ is a regular covering over a non-trivial graph manifold. Then the degree $d$ of $\phi$ satisfies the equation

$$
d \sum_{p \in I_{i}} \sum_{q \in I_{j}} \tilde{b}_{p q}=b_{i j}\left|I_{i}\right|\left|I_{j}\right| .
$$

where $\left(b_{i j}\right)\left(\left(\tilde{b}_{p q}\right)\right)$ is the B-matrix of $M(\tilde{M})$. 


\section{2. $B$-matrix, $H$-matrix and covering degrees}

Let $M$ be a non-trivial graph manifold. The symmetry of the $B$-matrix $B(M)$ will provide some useful information in determining the covering degrees. To clarify the idea, a reduced weighted graph $L(M)$ is defined in [WW, §9] as follows. The graph $L(M)$ has the same vertices as $\Gamma(M)$, and it has one edge $e_{i j}$ for each pair of vertices $v_{i}, v_{j}$ ( $i$ may be equal to $j$ ). Assign $b_{i j}$ as the weight of $e_{i j}$.

An isometry $g$ of $L(M)$ is an automorphism of the graph which preserves the weights. That is, the weight of $e_{i j}$ equals that of $g\left(e_{i j}\right)$ for all $i, j$. Denote the isometry group of $L(M)$ by $G(M)$. It induces an action on the set $V(M)$ of vertices of $L(M)$.

Suppose $\phi: \tilde{M} \rightarrow M$ is a regular covering map. A covering transformation $f: \tilde{M} \rightarrow \tilde{M}$ induces a map $f_{\#}: L(\tilde{M}) \rightarrow L(\tilde{M})$ which is clearly an isometry. Let $F(\tilde{M})$ be the set of isometries induced by covering transformations. Then $F(\tilde{M})$ is a subgroup of $G(\tilde{M})$. Since $\phi$ is a regular covering, it induces an one-to-one correspondence $\mathrm{V}(\tilde{M}) / \mathrm{F}(\tilde{M}) \rightarrow V(M)$. Since $F(\tilde{M})$ is a subgroup of $G(\tilde{M})$, for each vertex $v \in V(M), \phi^{-1}(v)$ is contained in a $G$-orbit of $V(\tilde{M})$.

Suppose $V(\tilde{M})$ has $m$ disjoint $G$-orbits $\tilde{U}_{1}, \tilde{U}_{2}, \cdots, \tilde{U}_{m}$, and $V(M)$ has $n$ vertices $v_{1}, v_{2}, \cdots, v_{n}$. For each $G$-orbit $\tilde{U}_{\alpha}$ of $V(\tilde{M})$, define its indexing set

$$
O_{\alpha}=\left\{r \mid \tilde{v}_{r} \in \tilde{U}_{\alpha}\right\}
$$

Each $G$-orbit of $V(\tilde{M})$ is the union of some $\phi^{-1}(v), v \in V(M)$. Define

$$
A_{\alpha}=\left\{i \mid I_{i} \subset O_{\alpha}\right\}
$$

Then we have

$$
O_{\alpha}=\bigcup_{i \in A_{\alpha}} I_{i}
$$

We use $\left|O_{\alpha}\right|$ to denote the number of elements in $O_{\alpha}$, then we have $\left|O_{\alpha}\right|=$ $\sum_{i \in A_{\alpha}}\left|I_{i}\right|$.

Define

$$
\tilde{h}_{\alpha \beta}=\frac{1}{\left(\left|O_{\alpha}\right|\left|O_{\beta}\right|\right)} \sum_{r \in O_{\alpha}} \sum_{s \in O_{\beta}} \tilde{b}_{r s}, \alpha, \beta=1 \cdots m
$$

Then $\tilde{H}=H(\tilde{M})=\left(\tilde{h}_{\alpha \beta}\right)_{m \times m}$ is a non-negative symmetric matrix determined by $L(\tilde{M})$ the indexing of $G$-orbit of $\tilde{V}(M)$.

Definition 5. $\tilde{H}=H(\tilde{M})$ is called the $H$-matrix of $\tilde{M}$, for a non-trivial graph manifold $\tilde{M}$.

Lemma 2. Suppose $\tilde{M}$ is a connected non-trivial graph manifold. Then each column (row) of $H(\tilde{M})$ has at least one non-zero element. 
Proof. Since $\tilde{M}$ is connected, each column (row) of $B(\tilde{M})$ has at least one non-zero element. Then Lemma 2 follows from the definition of $H(\tilde{M})$.

Theorem 2. Suppose $\phi: \tilde{M} \rightarrow M$ is a d-fold regular covering over a non-trivial graph manifold. Then d satisfies the equation

$$
d \tilde{h}_{\alpha \beta}\left|O_{\beta}\right|=\sum_{j \in A_{\beta}} b_{i j}\left|I_{j}\right| \quad \text { for any } \alpha, \beta=1 \cdots m, \quad i \in A_{\alpha} .
$$

Proof. For any $\tilde{v}_{p}, \tilde{v}_{q} \in \tilde{U}_{\alpha}$, there is an isometry $g \in G(\tilde{M})$ sending $\tilde{v_{p}}$ to $\tilde{v_{q}}$. Suppose $g$ maps $\tilde{v}_{s}$ to $\tilde{v}_{g(s)}$ for $\tilde{v}_{s} \in \tilde{U}_{\beta}$. Since $g$ is an isometry, we have $\tilde{b}_{p s}=\tilde{b}_{q g(s)}$. Since $\tilde{U}_{\beta}$ is a $G$-orbit, when $\tilde{v}_{s}$ ranges over all vertices of $\tilde{U}_{\beta}, \tilde{v}_{g(s)}$ also ranges over all vertices of $\tilde{U}_{\beta}$. Therefore, we have

$$
\sum_{s \in O_{\beta}} \tilde{b}_{p s}=\sum_{s \in O_{\beta}} \tilde{b}_{q g(s)}=\sum_{s \in O_{\beta}} \tilde{b}_{q s}=C_{\alpha \beta}
$$

$C_{\alpha \beta}$ is a constant determined by $\alpha$ and $\beta$, and

$$
C_{\alpha \beta}=\frac{1}{\left|O_{\alpha}\right|} \sum_{r \in O_{\alpha}} \sum_{s \in O_{\beta}} \tilde{b}_{r s}=\tilde{h}_{\alpha \beta}\left|O_{\beta}\right|
$$

By Theorem 1, we have

$$
b_{i j}\left|I_{i}\right|\left|I_{j}\right|=d \sum_{r \in I_{i}} \sum_{s \in I_{j}} \tilde{b}_{r s}
$$

Assume $i \in A_{\alpha}$. When $j$ ranges over $A_{\beta},(2.6)$ becomes

$$
\begin{aligned}
\sum_{j \in A_{\beta}} b_{i j}\left|I_{i}\right|\left|I_{j}\right| & =d \sum_{j \in A_{\beta}} \sum_{r \in I_{i}} \sum_{s \in I_{j}} \tilde{b}_{r s} \\
& =d \sum_{r \in I_{i}} \sum_{s \in O_{\beta}} \tilde{b}_{r s} \\
& =d\left|I_{i}\right| . C_{\alpha \beta} \\
& =d\left|I_{i}\right| \tilde{h}_{\alpha \beta}\left|O_{\beta}\right| .
\end{aligned}
$$

The last three equations follow from (2.1) (2.4) and (2.5). Then (2.3) follows.

To express Theorem 2 in terms of matrix equation, we have the following theorem. 
Theorem 3. Suppose $\phi: \tilde{M} \rightarrow M$ is a d-fold regular covering over a non-trivial graph manifold. Then d satisfies the equation:

$$
X_{m \times n} B_{n \times n}=d \tilde{H}_{m \times m} W_{m \times n} .
$$

where $B$ is the $B$-matrix of $M$, and $\tilde{H}$ is the $H$-matrix of $\tilde{M}, X$ and $W$ satisfy:

(a) both $X$ and $W$ are $m \times n$ non-negative matrices;

(b) each column of $X$ has only one non-zero element and the row sums of $X$ are constant 1 ;

(c) $W$ is obtained from $X$ by substituting the only non-zero element in each column of $X$ for 1 . Then the column sums of $W$ are constant 1.

Proof. Define

$$
\begin{gathered}
x_{\alpha i}=\left\{\begin{array}{l}
\frac{\left|I_{i}\right|}{\left|O_{\alpha}\right|} \text { if } i \in A_{\alpha}, \\
0 \text { if } i \notin A_{\alpha} .
\end{array}\right. \\
w_{\alpha i}=\left\{\begin{array}{l}
1 \text { if } i \in A_{\alpha}, \\
0 \text { if } i \notin A_{\alpha} .
\end{array}\right.
\end{gathered}
$$

We denote $X=\left(x_{\alpha i}\right), W=\left(w_{\alpha i}\right)$. Clearly in the $\alpha$-th row of $X, x_{\alpha i} \neq 0$ if and only if $i \in A_{\alpha}$. Since $\left|O_{\alpha}\right|=\sum_{i \in A_{\alpha}}\left|I_{i}\right|$, we have the row sums of $X$ are constant 1 and each column of $X$ has only one non-zero element. So (b) is true and $W$ is determined by $X$ just like (c).

Assume that $i \in A_{\alpha}$, by (2.9), (2.3) and the symmetry of $B$, we have

$$
(X B)_{\beta i}=\sum_{j \in A_{\beta}} x_{\beta j} b_{j i}=\sum_{j \in A_{\beta}} \frac{\left|I_{j}\right|}{\left|O_{\beta}\right|} b_{j i}=d \tilde{h}_{\alpha \beta}
$$

By (2.10) and the symmetry of $\tilde{H}$ we have

$$
(d \tilde{H} W)_{\beta i}=d \sum_{k=1}^{m} \tilde{h}_{\beta k} w_{k i}=d \tilde{h}_{\beta \alpha}=d \tilde{h}_{\alpha \beta}
$$

So we got the equation (2.8) of Theorem 3 .

\section{Nontrivial graph manifolds have Property C}

The following Lemma will be used in proving our main result.

Lemma 3. ([HJ, Lemma 8.1.21 and Theorem 8.3.1.]) If $A=\left(a_{i j}\right)$ is a $m \times m$ non-negative matrix, satisfies the column (row) sums are constant 1 , then the 
maximum eigenvalue of $A$ is 1 , and there is an non-negative eigenvector $x=$ $\left(x_{1}, \cdots, x_{m}\right)^{T}, x \neq 0, x_{i} \geq 0, i=1 \cdots m$ such that $A x=x$.

Theorem 4. Suppose $\phi, \phi^{\prime}: \tilde{M} \rightarrow M$ are regular coverings over a non-trivial graph manifold with covering degrees $d, d^{\prime}$. Then $d=d^{\prime}$. That is, a non-trivial graph manifold has Property $C_{r}$.

Proof. By Theorem 3 we have the following matrix equations.

$$
\left\{\begin{array}{l}
\left(X_{1}\right)_{m \times n} B_{n \times n}=d \tilde{H}_{m \times m}\left(W_{1}\right)_{m \times n} \\
\left(X_{2}\right)_{m \times n} B_{n \times n}=d^{\prime} \tilde{H}_{m \times m}\left(W_{2}\right)_{m \times n}
\end{array} .\right.
$$

Then we have

$$
\left\{\begin{array}{l}
X_{1} B X_{2}^{T}=d \tilde{H} W_{1} X_{2}^{T} \\
X_{2} B X_{1}^{T}=d^{\prime} \tilde{H} W_{2} X_{1}^{T}
\end{array}\right.
$$

Since both $B$ and $\tilde{H}$ are symmetric matrices, we have

$$
d \tilde{H} W_{1} X_{2}^{T}=\left(d^{\prime} \tilde{H} W_{2} X_{1}^{T}\right)^{T}=d^{\prime} X_{1} W_{2}^{T} \tilde{H}
$$

Denote

$$
L=X_{1} W_{2}^{T}, \quad R=W_{1} X_{2}^{T}, \quad \lambda=\frac{d}{d^{\prime}}
$$

then we have

$$
L \tilde{H}=\lambda \tilde{H} R
$$

Recall both $X_{i}$ and $W_{i}$ are $m \times n$ non-negative matrices, which satisfy the conditions (b) and (c) in Theorem 3 : the row sums of $X_{i}$ and the column sums of $W_{i}$ are both constant 1 . Then it is easy to see both $L$ and $R$ are non-negative matrices, the row sums of $L$ and column sums of $R$ are both constant 1 . (If we denote $J_{m}=\underbrace{(1 \cdots 1)^{T}}_{\text {m times }}, J_{n}=\underbrace{(1 \cdots 1)^{T}}_{\mathrm{n} \text { times }}$, then we have $L J_{m}=X_{1} W_{2}^{T} J_{m}=X_{1} J_{n}=$ $\left.J_{m}, R^{T} J_{m}=X_{2} W_{1}^{T} J_{m}=X_{2} J_{n}=J_{m}\right)$.

By Lemma 3, the maximum eigenvalues of $L$ and $R$ are both 1 , and there is non-negative vector $y=\left(y_{1}, \cdots, y_{m}\right)^{T}$ satisfies

$$
R y=y, \quad y \neq 0, \quad y_{i} \geq 0 \quad(i=1, \cdots, m) .
$$

By (3.4) and (3.5), we have

$$
L(\tilde{H} y)=\lambda(\tilde{H} y)
$$

By Lemma 2, each column (row) of $\tilde{H}$ has at least one non-zero element. Since $\tilde{H}$ is a non-negative matrix, $y$ is a non-negative vector and $y \neq 0$, it follows that $\tilde{H} y \neq 0$. So $\lambda$ is the eigenvalue of $L$ by (3.6). The maximum eigenvalue of $L$ is 
1 , so we must have $\lambda \leq 1$. That is, $d \leq d^{\prime}$. By the symmetry of $\phi$ and $\phi^{\prime}$, we conclude $d^{\prime} \leq d$ also. Hence $d=d^{\prime}$

By Theorem 4 and [WW, Lemma 2.1], we have

Corollary 5. Every non-trivial graph manifold has Property $C$.

By Corollary 5, [WW, Theorem 2.5] and [WW, Theorem 8.6], we have

Corollary 6. A geometric 3-manifold has Property $C$ if and only if it is not covered by either (surface) $\times S^{1}$ or a torus bundle over $S^{1}$. In particular, Conjecture 1 is true.

\section{Property $I$ of 3-manifold groups}

A group $G$ is called cohopfian if each self-monomorphism of $G$ is an isomorphism. Recently when 3-manifolds groups are cohopfian have been studied (see [GW], $[\mathrm{WW}])$.

Definition 6. A group $G$ has property $I$ if, whenever $G_{1}$ and $G_{2}$ are isomorphic subgroups of $G$, then their embedding indices either are the same or are both infinite.

Remark 2. Both Property $C$ and cohopficity are related indices of embeddings one group to another, the first one restricted on finite index embeddings and the second one restricted on self-embeddings. The notion Property $I$ unifies two notions Property $C$ and cohopficity.

Theorem 7. Suppose $M$ is a closed geometric 3-manifold. Then the fundamental group $\pi_{1}(M)$ has Property I if and only if $M$ is irreducible and $M$ is not covered by either $($ surface $) \times S^{1}$ or a torus bundle over $S^{1}$.

Proof. One direction follows directly from [WW, Theorem 8.7] and Remark 2.

Now we prove another direction. Denote $G=\pi_{1}(M)$, where $M$ is irreducible and it is not covered by either (surface) $\times S^{1}$ or a torus bundle over $S^{1}$. Then $M$ has property $C$ by Corollary 7. $M$ is aspherical $[\mathrm{J}]$, and therefore for any covering $\tilde{M}$ of $M, H_{3}\left(\pi_{1}(\tilde{M})\right)=H_{3}(\tilde{M})$.

Let $G_{1}$ and $G_{2}$ be the isomorphic subgroup of $G$. Let $\tilde{M}_{1}$ and $\tilde{M}_{2}$ be the corresponding coverings. Then we have

$$
H_{3}\left(\tilde{M}_{1}\right)=H_{3}\left(\pi_{1}\left(\tilde{M}_{1}\right)\right)=H_{3}\left(G_{1}\right)=H_{3}\left(G_{2}\right)=H_{3}\left(\pi_{1}\left(\tilde{M}_{2}\right)\right)=H_{3}\left(\tilde{M}_{2}\right) .
$$

Now $H_{3}\left(\tilde{M}_{i}\right)=Z$ if and only if $\tilde{M}_{i}$ is closed, and this is true if and only if 
$\left[G, G_{i}\right]$ is finite. So either both $\left[G, G_{1}\right]$ and $\left[G, G_{2}\right]$ are infinite, or both $\left[G, G_{1}\right]$ and $\left[G, G_{2}\right]$ are finite. In the later case, if $G$ is finite, clearly $\left[G, G_{1}\right]=\left[G, G_{2}\right]$. If $G$ is infinite, then and $\tilde{M}_{i}$ is either a closed hyperbolic manifold, or a closed Haken manifold, or a closed Seifert manifold of infinite $\pi_{1}$. Since $\tilde{M}_{1}$ and $\tilde{M}_{2}$ have isomorphic fundamental groups, they are homeomorphic [T]. Since $M$ has property $C$, the two covering degrees are the same. Hence $\left[G, G_{1}\right]=\left[G, G_{2}\right]$.

\section{References}

[GW] F. Gonzales-Acuna and W. Whitten, Embeddings of 3-manifold groups, Mem. Amer. Math. Soc. 474 (1992),

[HJ] R.A.Horn and C.R.Johnson, Matrix analysis, Cambridge University Press, 1985.

[J] W. Jaco, Lectures on Three manifold Topology, Conference board of the Mathematical Sciences, AMS 1977.

[K] R. Kirby, Problems in low dimensional manifold theory, Algebraic and geometric topology (ed. R. J.Milgram). In: Proceedings of Symposia in Pure Mathematics 32, American Mathematical Society, Providence, R.I. 1978, pp. 273-312.

[LW] J. Luecke and Y-Q. Wu, Relative Euler number and finite covers of graph manifolds, In: Proceedings of the Georgia International Topology Conference, AMS/IP Stusies in Advanced Mathematics Vol.2 (Part 1) 1997, pp. 80-103.

[N] W.D. Neumann, Commensurability and virtual fibration for graph manifolds, Topology 36 No. 2 (1997), 355-378.

[S] P. Scott, The geometries of 3-manifolds, Bull. London. Math. Soc. 15 (1983), 401-487.

[T] W. Thurston, Three dimensional manifolds, Kleinian groups and hyperbolic geometry, Bull. Amer. Math. Soc. 6 (1982), 357-382.

[WW] S.C. Wang and Y.Q. Wu, Covering invariant of Graph manifolds and cohopficity of 3manifold groups, Proc. London Math. Soc. 68 (1994), 221-242.

[WY] S.C. Wang and F.C. Yu Graph manifolds with non-empty boundary are covered by surface bundles, Math. Proc. Camb. Phil. Soc. 122 (1997), 447-455.

Fengchun Yu and Shicheng Wang

Department of Mathematics

Peking University

Beijing 100871

China

e-mail:swang@sxx0.math.pku.edu.cn

(Received: May 6, 1996) 